\title{
Stylized facts of financial markets and market crashes in Minority Games
}

\author{
Damien Challet ${ }^{(1)}$, Matteo Marsili ${ }^{(2)}$ and Yi-Cheng Zhang ${ }^{(3)}$ \\ (1) Theoretical Physics, Oxford University, 1 Keble Road, Oxford OX1 3NP, United Kingdom \\ (2) Istituto Nazionale per la Fisica della Materia (INFM), Unitá di Trieste-SISSA, I-34014 Trieste, Italy \\ (3) Institut de Physique Théorique, Université de Fribourg, Perolles CH-1700, Switzerland
}

(October 23, 2018)

\begin{abstract}
We present and study a Minority Game based model of a financial market where adaptive agents - the speculators - interact with deterministic agents - called producers. Speculators trade only if they detect predictable patterns which grant them a positive gain. Indeed the average number of active speculators grows with the amount of information that producers inject into the market. Transitions between equilibrium and out of equilibrium behavior are observed when the relative number of speculators to the complexity of information or to the number of producers are changed. When the system is out of equilibrium, stylized facts arise, such as fat tailed distribution of returns and volatility clustering. Without speculators, the price follows a random walk; this implies that stylized facts arise because of the presence of speculators. Furthermore, if speculators abandon price taking behavior, stylized facts disappear.
\end{abstract}

\section{INTRODUCTION}

Physicists' interest for Economy and more specifically financial markets has exploded in last two years. An important part of the research is the analysis of financial data [1] 3], which has led to the characterization of some empirical statistical regularities, known as "stylized facts". Consequently a lot of work have attempted to build models of markets that reproduce these properties in order to understand their cause. A very promising way is to consider agents based models [4.5. However, in spite of interesting results obtained so far by numerical simulations, most of these models are too complex and not well suitable to an analytic approach that could explain the origin of their complex behavior.

A different strand of literature, originated by the introduction of the Minority Game (MG) [6], has instead focused on highly simplified toy models of financial markets . On one hand, variants of this model have been shown to reproduce quite accurately the stylized facts of financial markets 8 11]. On the other, there are analytic approaches for this model which provide exact results for the limit of infinitely many agents $12[16]$. These approaches give a coherent picture of the collective properties and allow one to investigate in detail a number of issues on the behavior of complex systems of interacting agents, such as the role of market impact [13, 15$]$ and the interplay between different types of agents in a market [16.

Our aim is to present what we believe to be the simplest MG giving rise to a quite complex and rich behavior. In different regions of its phase space, the model de-

\footnotetext{
${ }^{*}$ See [1] for a large collection of commented references about the $\mathrm{MG}$.
}

scribes markets with gaussian statistics and short ranged correlation, markets with fat tailed retuns and long range correlations and even market crashes. In addition, it is possible to obtain exact analytical results 17] along the lines of refs. 12,13.

The first key ingredient of the model is the interplay between two types of traders. The first type, called producers in ref. 18, 16], are traders who use the market for exchanging goods; Their trading decisions originate from outside opportunities related to the economic activity and not on the market dynamics itself. These traders have a predictable behavior with respect to a news arrival process and hence they inject information into the market. They represent the underlying economic activity, so they could also be called "fundamentalists": in the absence of other types of agents market prices would follow a random walk, which we could call the fundamentals.

The other type of traders are speculators. They are adaptive agents with bounded rationality. They study the relationship between the news arrivals and market reactions in order to anticipate market movements. Their aim is to gain from market fluctuations. On one hand they provide liquidity for producers, on the other they color the white noise process produced by the latter and by the news arrival process. Therefore they are responsible for the emergence of stylized facts.

The second key ingredient of the model is that we allow the speculators for the possibility of not trading if the market does not contain sufficiently profitable arbitrage opportunities for them. In the language of Physics, this makes the model grand canonical. Such models have been studied in refs. [8,9] and more recently in ref. [11], where a fundamental mechanism for long-range correlation of the volatility was proposed. However, the proposed grand canonical mechanism in refs [8,11] does not account for the risky nature of markets, by contrast to that we propose here, whereas the one found in ref [9] is 
in essence the one we consider here $\mathrm{I}$.

These two features - the interplay between producers and speculators and the possibility of not trading for speculators - have been introduced in ref. [16], though their consequences have not been fully exploted. In addition, the grand canonical mechanism proposed here is different and leads to qualitatively different results.

\section{THE MODEL}

We consider a set of agents who interact repeatedly in a market. In each period $t=1,2, \ldots$, each agent $i$ chooses an action $a_{i}(t)$, which is a real number. To fix ideas one may think that $a_{i}(t)>0$ means that agent $i$ wants to buy $a_{i}(t) \$$ of an asset whereas $a_{i}(t)<0$ implies that he wants to sell.

\section{A. Market mechanism and information}

Following ref. [10], we leave details at this level unspecified, and directly define the excess demand $A(t)=$ $\sum_{i=1}^{N} a_{i}(t)$ and the payoffs of agents:

$$
g_{i}(t)=-a_{i} A(t)
$$

These payoffs are such that those agents who are in "minority" $\left(a_{i} A(t)<0\right)$ are rewarded. This captures the fact that when there are a lot of buyers, sellers may sell at a higher price.

Following Refs. [8,5, 19,20], we define a price dynamics in terms of the excess demand, as

$$
\log p(t+1)=\log p(t)+r(t)=\log p(t)+\frac{A(t)}{\lambda}
$$

where $r(t)$ is the return at time $t$ and $\lambda$ is related to the market depth. The market is also characterized by a news arrival process which is modeled by an integer $\mu(t)$ which is drawn randomly [21,22] and independently in each period from the integers $1, \ldots, P . \mu(t)$ labels the "state of the world" which encodes all relevant economic information.

\section{B. Producers}

The first type of traders - the producers - behave in a deterministic way with respect to $\mu(t)$. This means that if $i$ is a producer, $a_{i}(t)$ is a function of $\mu(t)$ only,

\footnotetext{
${ }^{\dagger}$ Note however that it is not motivated by the risky nature of markets. In particular, we do not have to introduce utility functions.
}

i.e. $a_{i}(t)=\sigma_{i}^{\mu(t)}$. For each agent $i$ and state $\mu$ we draw randomly $\sigma_{i}^{\mu}$ from a fixed distribution. We shall take the bimodal distribution $\sigma_{i}^{\mu}= \pm 1$ with equal probability, in what follow:

Let $N_{p}$ be the number of producers and we introduce the reduced number $n_{p}=N_{p} / P$ for convenience.

\section{Speculators}

The second type of traders - the speculators - are adaptive. They are assigned a number $S+1$ of trading strategies: When speculator $i$ uses strategy $s=0,1, \ldots, S$, then his action is $a_{i}(t)=\sigma_{s, i}^{\mu(t)}$. For strategies $s>0-$ called active,$- \sigma_{s, i}^{\mu}$ is again drawn randomly and independently for each $s, i$ and $\mu$ from the bimodal distribution. For $s=0$, instead, $\sigma_{0, i}^{\mu}=0$ for all $\mu$.

In other words, producers can be regarded as speculators with just one active trading strategy and speculators can decide not to trade if they resort to their 0-strategy. At odd with producers, speculators have an additional degree of freedom, which is the choice of the strategy $s_{i}(t)$ they will play at time $t$. In order to take this decision, each speculator $i$ keeps track of the performance of each of his trading strategy $s$ by assigning a score $U_{i, s}$ to it. The strategy $s_{i}(t)$ which agent $i$ follows at time $t$, is that with the highest score. His action will then be $a_{i}(t)=\sigma_{i, s_{i}(t)}^{\mu(t)}$. The scores are updated according to

$$
U_{i, s}(t+1)=U_{i, s}-a_{i, s}^{\mu(t)} A(t)+\epsilon \delta_{s_{i}(t), 0} .
$$

This means that active strategies $s>0$ are "rewarded" by the (virtual) gain they would have given to agent $i$ if they had been played The $s=0$ strategy is instead rewarded by a constant amount $\epsilon>0$. This implies that an agent is willing to use a trading strategy only if it gives an average gain larger than $\epsilon$. One can interpret $\epsilon>0$ as modeling either a risk-free asset which ensures a constant gain - the bank interest rate - or more simply a riskpremium for not trading. The latter may reflects agent's risk aversion to trading in a risky market. Note that with $\epsilon<0$ and large enough, one recovers the standard MG because all agents use active strategies.

There are $N_{s}$ speculators and we find it convenient to introduce the reduced number $n_{s}=N_{s} / P$. Note that

\footnotetext{
${ }^{\ddagger}$ Any distribution with zero average and unit variance leads to exactly the same results in the limit $N \rightarrow \infty$.

${ }^{\S}$ This is true only if $A(t)$ would not have changed if $i$ had actually played strategy $s$ instead of $s_{i}(t)$ (which is why we speak of virtual gain). In other words, we are assuming that agents neglect their market impact and behave as price takers. This is by no means an innocent assumption as shown in refs. $12,13,15$ (see later).
} 
$n_{s}=1 / \alpha$ where $\alpha$ is the control parameter used in refs. 24, 12, 13 .

\section{RESULTS}

We performed extensive numerical simulations of the market model. For $P \gg 1$ all (intensive) quantities in the stationary state only depend on the parameters $n_{s}$, $n_{p}$ 24, 23, 12, 16] and $\epsilon$. All simulations were performed with $S=2$. As usual no qualitative change is expected for $S>2$ [13].

Before passing to the discussion of results, let us mention that the model can be solved along the lines of ref. [13]. An account of this solution will be presented in a forthcoming publication [17].

\section{A. Market's ecology}

The interplay between the two types of agents is shown in Fig. 1 for $\epsilon=-\infty$ (no 0-strategies): keeping the number of speculators fixed and increasing the number of producers, the market predictability

$$
H=\frac{1}{P} \sum_{\mu=1}^{P}\langle A \mid \mu\rangle^{2}
$$

increases ${ }^{\text {网 }}$ (left panel). When speculators are added again to the market (right panel) they exploit predictability and hence reduce it.

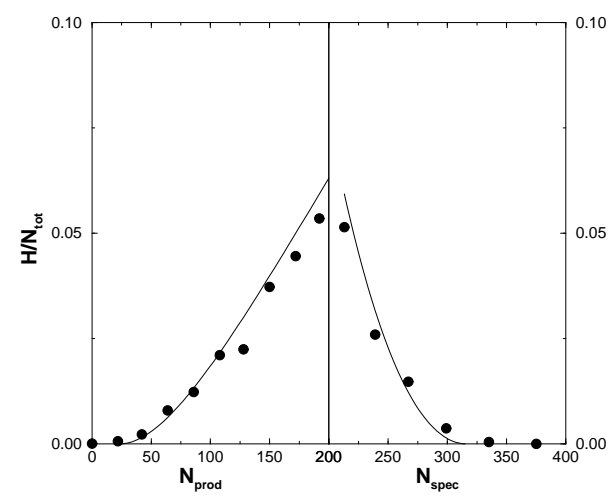

\footnotetext{
${ }^{* *}$ Here $\langle A \mid \mu\rangle$ is the average of $A(t)$ conditional on $\mu(t)=\mu$. If this quantity is non-zero, then the market is statistically predictable. $H$ measures predictability and is related to negative entropy.
}

FIG. 1. $N_{p}$ Producers are added to a standard MG ( $P=64, N=200, S=2)$ where the outcome is unpredictable $(H=0)$ : information content, or negative entropy, increases with $N_{p}$. Then, while $N_{p}$ is kept fixed at 200, additional speculators are added; they finally remove completely the information put by producers (average over 100 realizations). The continuous lines are analytical results from the exact solution.

The same behavior applies for $\epsilon>0$. For a fixed (reduced) number $n_{p}$ of producers, $H$ decreases as the number of speculators increases (see Fig. 2). As in the standard MG [23, 12], there is a phase transition separating a symmetric phase $(H=0)$, for $n_{s}>n_{s}^{*}\left(n_{p}\right)$, and an asymmetric phase $(H>0)$. The main difference is that in the symmetric phase many speculators refrain from playing: The number of active speculators $n_{s}^{\text {act }}$ saturates to a finite value as the number of speculators increases. The behavior of $n_{s}^{\text {act }}$ is characterized by a cusp at $n_{s}^{*}$ (see Fig. 2) which becomes more pronounced as $\epsilon \rightarrow 0^{+}$.

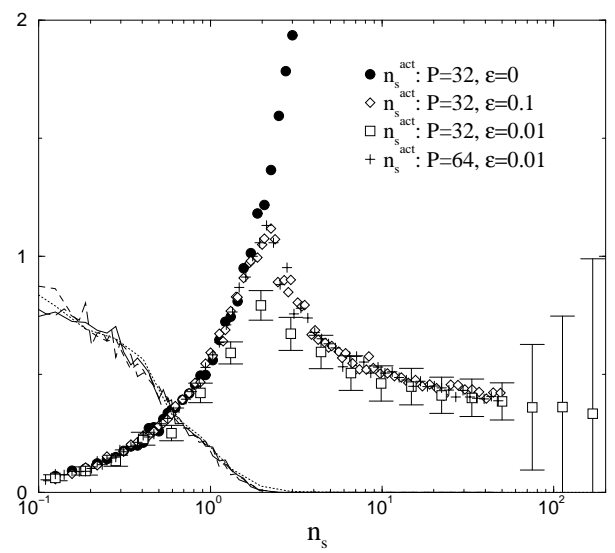

FIG. 2. Predictability per agent $H /\left(N_{p}+N_{s}\right)$ (lines) and number of active speculators $n_{s}^{\text {act }}$ (symbols) as a function of $n_{s}$ for $n_{p}=1$ and some values of $\epsilon$ and $P$. The phase transition point $n_{s}^{*}$ where $H \rightarrow 0$ and $n_{s}^{\text {act }}$ shows a cusp, does not depend on $\epsilon$. For $n_{s}>n_{s}^{*}, n_{s}^{\text {act }}$ has a discontinuous behavior as $\epsilon \rightarrow 0$. Indeed $n_{s}^{\text {act }}$ increases linearly with $n_{s}$ for $\epsilon \leq 0$ whereas it saturates to a finite value for $\epsilon>0$.

In the symmetric phase $n_{s}>n_{s}^{*}$, the market is kept marginally efficient in a dynamic way, that is, the market is efficient in the long run (i.e. $H=0$ ), while locally in time, it may be not efficient. Consequently, a fraction of speculators alternate periods of activity, in which he trades, and inactivity, in which he just watches the market, waiting for more favorable times. The level $n_{s}^{\text {act }}$ of activity of speculators is just barely sufficient to exploit the information injected into the market by producers, thus making the market efficient. Fig. 3 shows that the number $n_{s}^{\text {act }}$ of active speculators vanishes in the absence of producers and it increases as $n_{s}^{\text {act }} \sim \sqrt{n_{p}}$ as the number of producers increases: this is also the quantity of information put into the market by the producers and asserts the validity of the proposed fundamental grand- 
canonical mechanism.

The phase diagram of the symmetric phase with respect to $n_{s}, n_{p}$ and $\epsilon$ is quite complex, hence will be studied in details in a forthcoming publication, but let us discuss roughly its structure. At fixed $n_{s}$,

Again, the behavior for $\epsilon \leq 0$ is quite different: The activity does not vanish with $n_{p}$ but it rather remains finite even with $n_{p}=0$.

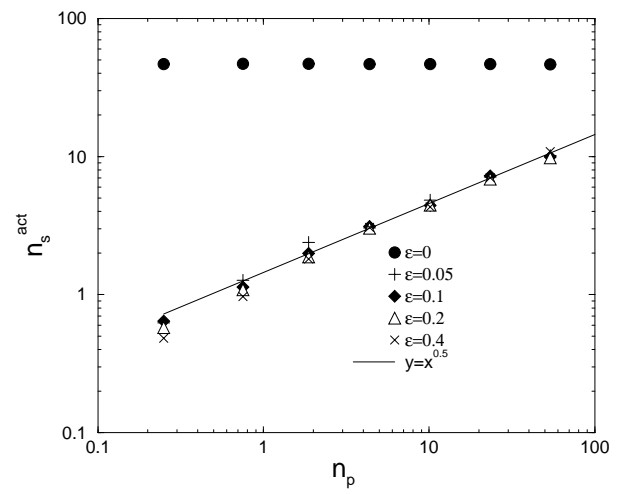

FIG. 3. Number of active speculators $n_{s}^{\text {act }}$ as a function of the number of producers $n_{p}$ for $P=4, n_{s}=62.5$ and several values of $\epsilon$.

\section{B. Market crashes}

Finally we remark that fig. 2 shows that the fluctuations of $n_{s}^{\text {act }}$ around its average diverge with $n_{s}$. This is due to the fact that as $n_{s}$ increases the stationary state which agents reach becomes more and more unstable. A snapshot of the dynamics deep in the symmetric phase is reported in fig. 4. The market repeatedly undergoes catastrophic events, i.e. crashes: Just after a crash, all speculators refrain from trading and $n_{s}^{\text {act }} \simeq 0$. This leaves the arbitrage opportunities created by producers unexploited. Then speculators gradually gain confidence and start trading again. The volume of speculation reaches a constant average value, but fluctuations of $n_{s}^{\text {act }}$ gradually increase. This process reaches a point when a large number of speculators start rushing into the market all at once. This causes violent shocks to the market which causes discontinuities in the price, i.e. crashest. The market crash drives speculators away from the market and the dynamics starts anew. The frequency of crashes increases with $n_{s}$ and it decreases with $\epsilon$.

In spite of its simplicity, the model provides a quite realistic picture of such a complex phenomenon as a market crash.

\footnotetext{
${ }^{\dagger \dagger}$ Given the symmetry of the model, positive jumps in $p(t)$ are as likely as negative ones.
}

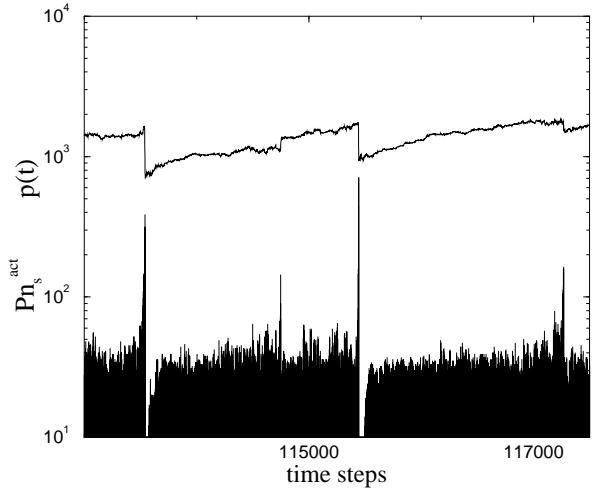

FIG. 4. Snapshot of the dynamics for $n_{s}=300, n_{p}=10$ $(P=32)$ and $\epsilon=0.01$. The price $p(t)$ (top) and speculators contribution to the volume $P n_{s}^{\text {act }}$ (bottom) is shown.

\section{STYLIZED FACTS}

\section{A. Volatility and volume clustering}

Volatility clustering is a very well known stylized fact. It is known that the volatility has algebraically decaying auto-correlation, and accordingly that the returns activity is clustered in time, which is an easy pattern to detect with naked eyes.

Fig. 5 illustrates this phenomenon for the present model (we define the volatility at time $t$ as $\sigma(t)=|A(t)|$ ). The deeper one goes in the symmetric phase - i.e. the larger $n_{s}$ - the more high volatility regions appear clustered. Indeed market crashes occur more and more frequently as $n_{s}$ increases.

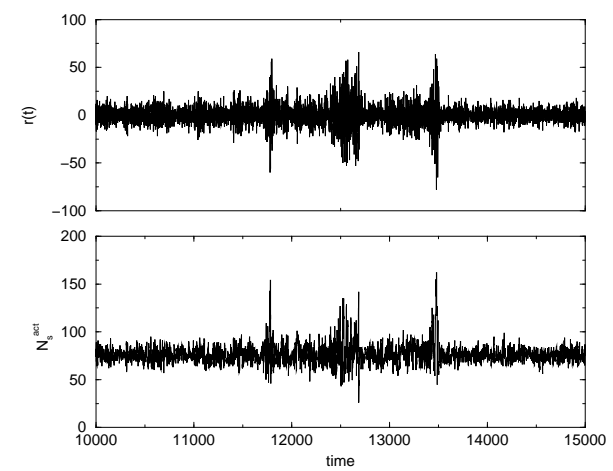

FIG. 5. Return and number of speculators in the market versus time. The volatility is clustered, as is the volume $\left(P=16, S=2, N_{s}=501, N_{p}=1001, \epsilon=0.01\right)$

The volatility auto-correlation is known to be algebraically decaying, typically as $\tau^{-0.3}$ in real financial markets [2]. Volatility auto-correlation is known to be related to volume correlation [2].

Figure 6 shows that the long ranged correlation of volatility occurs also in the present model, with an exponent that can be close to that of real markets: it depends quantitatively on the parameters $n_{s}, n_{p}$ and $\epsilon$ of 
the model, and exponents ranging from 0.09 up to 0.6 have already been observed, so that the let us only mention that this behavior is not universal, and can disappear if the parameters are extremal. A systematic quantitative study of this behavior in the whole phase space of the model, appears quite demanding and will be pursued elsewhere.

Note furthermore that the same behavior (with an exponent -0.65 ) was also found recently in the MG with evolving capitals [10]. This suggests that volatility clustering is a generic feature of financial markets and not an universal one, as also suggested recently in ref. [11.

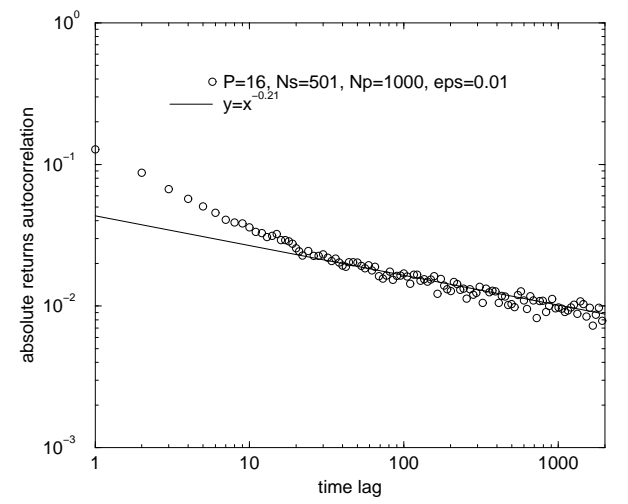

FIG. 6. Autocorrelation of the absolute returns $(P=16$, $\left.S=2, N_{s}=501, N_{p}=1000, \epsilon=0.01\right)$. The straight line as a -0.21 slope

\section{B. Return and volume histograms}

In real market, the probability distribution function (pdf) of returns is known to have fat tails with exponent -4 on average [1]. Figure 7 shows that the MG presented here can reproduce fat tail behavior. Note however that the value of the exponent depends on the parameters $n_{s}$, $n_{p}$ and $\epsilon$. For instance, the exponent of the tails decreases as $n_{s}$ increases at fixed $n_{p}$, i.e. the tails become fatter and fatter as the number of prospective speculators increases while the gain opportunities remain fixed用. The volume was also found to have a power-law tail distribution. Figure 7 shows typical returns histogram; parameter have been adjusted in order to obtain an exponent close to that of real markets [1].

\footnotetext{
${ }^{\ddagger \ddagger}$ We postpone the detailed study of this point to a forthcoming publication
}

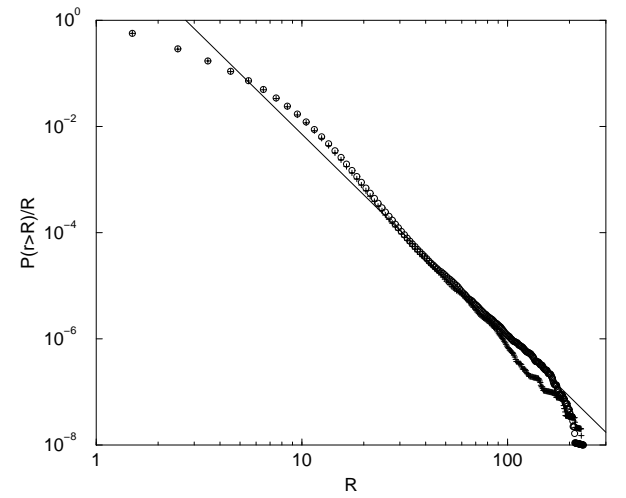

FIG. 7. Cumulative function of the returns $R$ divided by the return $R$ (circles: positive returns, x: negative returns) $\left(P=16, S=2, N_{s}=1001, N_{p}=1200, \epsilon=0.01\right) ;$ the continuous line has a slope of -3.8 , close to the one observed in financial markets.

\section{CONCLUSION}

The modified MG presented here is able to reproduce qualitatively a whole range of stylized facts. Most importantly, its quite rich behavior can be studied analytically 117 along the lines of refs. 12, 13]. At odds with the standard MG, speculators in this model market are extremely sensitive to the number of producers, and behave sensibly.

All the features discussed in the model crucially depends on the fact that agents neglect their market impact, i.e. behave as price takers. As soon as agents start to account even approximately for their market impact, as in refs. 13.15, the situation changes dramatically. In particular: i) the phase transition disappears, ii) the dynamics converges to one of exponentially many states where each speculator either plays one and the same strategy at all times, or he doesn't play at all, iii) volatility clustering and fat tailed distribution of returns disappear. The latter means, in particular, that all the stylized facts about financial markets crucially depends on the fact that agents behave as price takers.

These conclusions - strictly speaking- only hold in the highly simplified world described by our model. In real markets things are of course much more complex: agents trade at different frequencies, over different shares and with a large number of instruments such as derivatives; their importance varies greatly, from big investment funds and banks to small gamblers. Will the simplified picture, which results from our simple model, survive even when all these issues have been taken into account?

Partial positive answers have already been derived in recent work where, for example, the existence of a phase transition has been shown to persist when different types of agents are present [16], when agents have different weights [10] and when they trade at different frequencies [25]. Here we showed that a phase transition still persists 
when agents are allowed not to trade. At any rate, the present work opens a route to the understanding of how complex phenomena - such as volatility auto-correlation, fat tails in the pdf of returns and market crashes - may arise in financial markets. This route may not be unique, but it is definitely worth of investigation.

We acknowledge interesting discussions with Neil F. Johnson. This work has been supported in part by the Swiss National Funds for Scientific Research.

[1] V. Plerou, P. Gopikrishnan, L.. A. Nunes Amaral, X. Gabaix, H. E. Stanley, Phys. Rev. E. (Rapid Comm.) 62 (2000) R3023, preprint cond-mat/0008113

[2] P. Gopikrishnan, V. Plerou, X. Gabaix, H. E. Stanley, preprint cond-mat/0008113

[3] R. Mantegna and E. Stanley, Introduction to Econophysics, Cambridge University Press, 1999

[4] T. Lux and M. Marchesi, Nature 397, 498 - 500 (1999)

[5] G. Caldarelli, M. Marsili and Y.-C. Zhang, Europhys. Lett, 40 (5), pp. 479-484 (1997), cond-mat/9709118

[6] Challet D. and Zhang Y.-C., Physica A 246, 407 (1997) (adap-org/9708006.

[7] See the Minority Game's web page on http://www.unifr.ch/econophysics

[8] P. Jefferies, M.L. Hart, P.M. Hui, N.F. Johnson, Int. J. Th and Appl. Fin. 3-3 (2000), preprint condmat/9910072.

[9] T. S. Lo, P. M. Hui, N. F. Johnson, preprint condmat/0008387

[10] D. Challet, A. Chessa, M. Marsili, Y.-C. Zhang, Quant. Fin. I-1 (2001), preprint cond-mat/0011042

[11] J.-P. Bouchaud, I. Giardina, M. Mezard, preprint condmat/0012156

[12] D. Challet, M. Marsili and R. Zecchina, Phys. Rev. Lett 84, 1824 (2000), preprint cond-mat/9904392

[13] M. Marsili, D. Challet and R. Zecchina, Physica A 280, 522 (2000), preprint cond-mat/9908480

[14] J. A. F. Heimel, A. C. C. Coolen, preprint condmat/0012045

[15] M. Marsili and D. Challet, Adv. Compl. Sys., 3-I (2001), preprint cond-mat/0004376.

[16] Challet D., M. Marsili and Y.-C. Zhang, Physica A 276, 284 (2000) preprint cond-mat/9909265

[17] D. Challet, M. Marsili, F. Ricci-Tersenghi and R. Zecchina, in preparation.

[18] Y.-C. Zhang, Physica A 269, 30 (1999). (condmat/9901243

[19] J. D. Farmer, Comp. Fin., Nov-Dec (1999), pp. 26-39

[20] J.-P. Bouchaud, R. Cont, Eur. Phys. J. B 6 (1998) 4, 543-550, preprint cond-mat/9801279

[21] A. Cavagna, Phys. Rev. E 59, R3783 (1999)

[22] D. Challet and M. Marsili, Phys Rev. E 62, 1862 (2000), preprint cond-mat/0004196

[23] D. Challet and M. Marsili, Phys. Rev. E 60, 6271 (1999), preprint cond-mat/9904071
[24] Savit R., Manuca R., and Riolo R., Phys. Rev. Lett., 82(10), 2203 (1999), preprint adap-org/9712006.

[25] M. Piai and M. Marsili in preparation. 